\title{
An infrequent case of intussusception caused by gastrointestinal stromal tumor in an adult patient
}

\author{
Mehmet Mahir Fersahoglu, ${ }^{1}$ Ayse Tuba Fersahoglu, ${ }^{1}$ Nuriye Esen Bulut, ${ }^{1}$ \\ Burcu Seher Anil Narin, ${ }^{2}$ Sinan Tezer ${ }^{1}$ \\ ${ }^{1}$ Department of General Surgery, Fatih Sultan Mehmet Training and Research Hospital, Istanbul, Turkey \\ ${ }^{2}$ Department of Radiology, Fatih Sultan Mehmet Training and Research Hospital, Istanbul, Turkey
}

\begin{abstract}
Intussusception may occur anywhere in the gastrointestinal system. Unlike its idiopathic childhood counterpart, it is uncommon during adult life and a definitive cause is usually found; almost half of cases develop with malignancy. Gastrointestinal stromal tumors (GIST) originate from interstitial Cajal cells of the gastrointestinal tract. They more frequently occur in the stomach and small intestines, and often grow extraluminally, making it unlikely to cause an obstruction or bleeding. Presently described is an unusual instance of ileo-ileal intussusception due to GIST.
\end{abstract}

Keywords: Intussusception; stromal tumor.

$\mathrm{I}^{\mathrm{n}}$ ntussusception is an entity that develops as a telescopic intubation of a proximal intestinal segment into a distal segment, and rarely (1\%), may cause a mechanical obstruction in any region of the gastrointestinal system. In childhood, idiopathic etiology is typical, while in almost all adult patients, an etiological factor can be demonstrated. In nearly half of adult cases, intussusception develops secondary to a malignancy $[1,2]$. Symptoms of mechanical obstruction due to intussusception can occur acutely, or it may develop in an intermittent, chronic form secondary to spontaneous resolution of telescoped bowel segments and re-invagination over time. Gastrointestinal stromal tumors (GIST) originating from Cajal cells, which assume the task of a pacemaker in the interstitial area, may occasionally lead to intussusception [3]. Since GISTs have a tendency to grow into extraluminal space, they rarely invaginate.

Currently described is an infrequently encountered case of intussusception secondary to GIST, which represents $0.4 \%$ of all gastrointestinal tumors.

\section{CASE REPORT}

A 64-year-old male patient presented at the outpatient clinic of general surgery with complaints of abdominal pain, frequent belching, and weight loss for

Received: October 15, 2015 Accepted: December 08, 2015 Online: August 26, 2017

Correspondence: Dr. Mehmet Mahir FERSAHOGLU. Fatih Sultan Mehmet Egitim Arastirma Hastanesi, Genel Cerahi Klinigi, Istanbul, Turkey. 


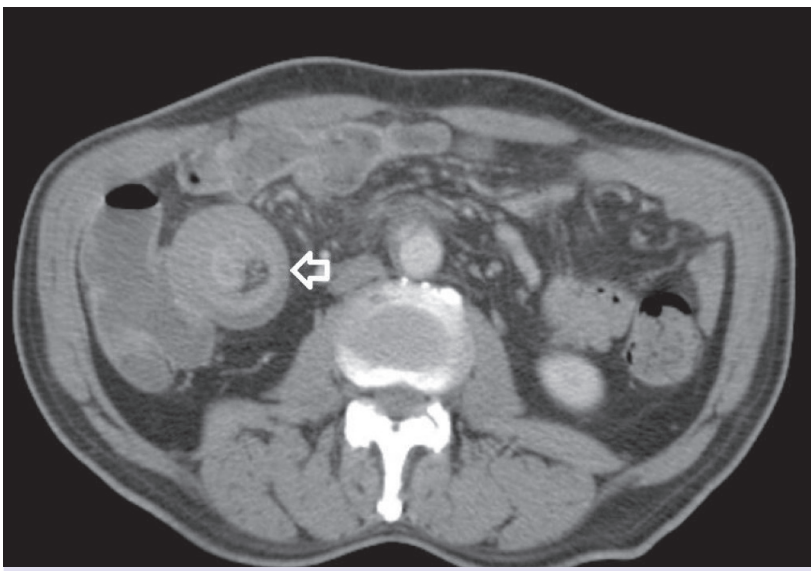

FIGURE 1. Computed tomography image of invaginated ileal segment.

previous 3 to 4 months. The patient indicated that he had occasional episodes of nausea without vomiting, but no problem with defecation. His personal history did not reveal any known disease or malignant disease in his family history. On physical examination, the only remarkable abdominal finding was minimal tenderness in the right lower quadrant. Digital rectal examination findings were also unremarkable. Serum biochemical and hematological parameters were within normal limits (white blood cell count: $7700 \mathrm{~K} / \mathrm{uL}$, hemoglobin: $12.8 \mathrm{~g} / \mathrm{dL}$, alanine aminotransferase: $10 \mathrm{IU} / \mathrm{L}$, aspartate aminotransferase: 13IU/L). Plain abdominal radiographs were unremarkable. Abdominal computed tomographs (CT) obtained to clarify reason for the tenderness localized in the lower abdominal quadrant revealed a mass lesion interpreted as plastron appendicitis in the area of the cecum or invagination (Figure 1). No abnormal finding was detected during colonoscopic or gastroscopic examination performed to detect intraluminal pathology. Laparoscopic exploration revealed invaginated bowel segments $30 \mathrm{~cm}$ proximal to the terminal ileum. However, due to the presence of diffuse adhesions, laparotomy was performed. The bowel segment was excised en bloc, and intestinal continuity was achieved with side-to-side ileo-ileal anastomosis (Figures 2,3). The postoperative period was uneventful and the patient was discharged on postoperative fifth day. After completion of histopathological analysis, the patient was diagnosed as GIST, and he is currently monitored by the oncology clinic (tumor diameter: $8 \times 5 \times 5 \mathrm{~cm}$; mitotic activity: decreased [5/50

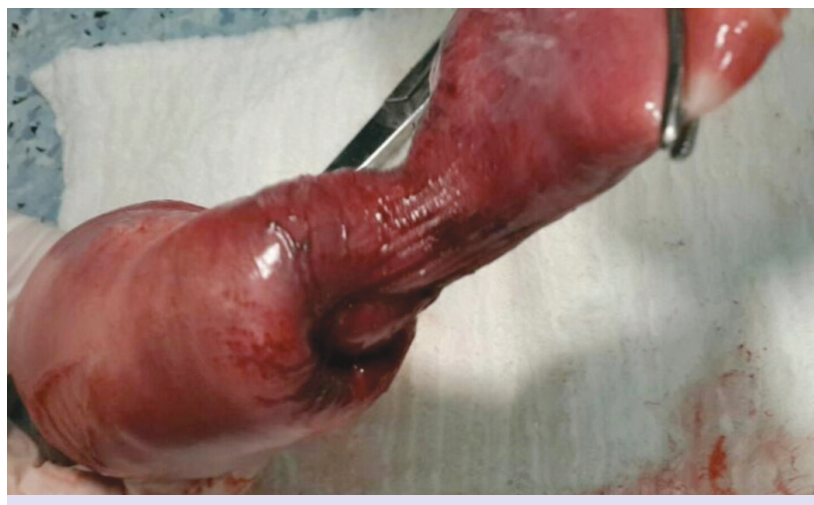

FIGURE2. Macroscopic appearance of invaginated ileal loop.

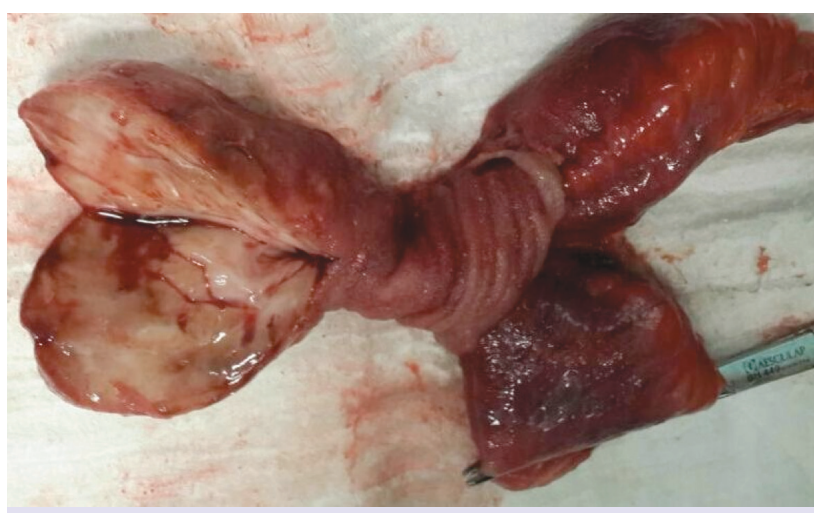

FIGURE 3. Stromal tumor that caused invagination.

BBA]; proto-oncogene c-Kit: $>50 \%++$ staining intensity. The lesion did not stain with CD34, smooth muscle actin, or desmin. S100: less than $10 \%$ of the cells were stained; $\mathrm{Ki}-67$ score: low [1\%]).

\section{DISCUSSION}

More than $90 \%$ of GISTs are seen in people aged $>40$ years. Although it is more frequently seen in men compared with women, incidence does not differ according to geographic region or ethnicity. [3]. It may cause bleeding or gastrointestinal symptoms. When it reaches a large size, in $20 \%$ of cases, intussusception can be seen, secondary to pain, palpable mass, and obstruction [4]. Intussusception that develops with the infolding of a proximal bowel segment into a distal segment is rarely seen in adult patients. Symptoms are typically nonspecific, including recurrent abdominal pain, nausea, and vomiting. In half of these patients, the lead point of telescopic infolding is malignant [2]. 
In nearly one-third of patients, preoperative diagnosis can be made based on history and physical examination combined with imaging modalities [5]. On ultrasonograph, telescopic multilayered intestinal wall may be visualized as a "bull's eye" or a "target sign." (Figure 1). Definitive diagnosis can be made based on intraoperative findings; however, oral or intravenous contrast-enhanced abdominal CT yields the most accurate result [6]. Usually, GISTs grow exophytically to the peripheral tissues and do not lead to the development of intussusception. However, since they retain the possibility -though rarely - like a pedunculated or sessile polyp, as was seen in this case, they can induce intussusception [7, 8]. Histological differentiation of GIST tumor from intestinal mesenchymal tumor can be achieved with CD117 staining or demonstration of the presence of proto-oncogene c-Kit. A newly developed oral formulation of a selective thyrosine kinase inhibitor with small molecular structure, imatinib, has enabled the prevention of recurrent GISTs during the postoperative period. Before the introduction of imatinib treatment, surgical excision was the only treatment modality, but recurrence rate remained high [9]. When adjuvant imatinib was administered to high-risk patients following surgical excision of GIST, a marked prolongation of survival time was observed when compared with placebo $[10,11]$. Based on these results, the use of imatinib as an adjuvant treatment in high-risk patients after surgical resection of GIST was approved by US Food and Drug Administration (FDA) in 2008, and the European Medicines Agency (EMA) in 2009. In a recent randomized Phase III study that included 400 high-risk GIST patients, imatinib treatment for 3 years provided statistically significant improvement over 1-year imatinib treatment (recurrence-free survival: $65.6 \%$ vs $47.9 \%$; $\mathrm{p}<0.001$ and overall survival: $92 \%$ vs $81.7 \%$; $\mathrm{p}<0.02$ ) [12]. Based on these outcomes, concurrently, both the FDA and the EMA advised that adjuvant treatment be maintained for 36 months in high-risk patients. Since spread to regional lymph nodes is rarely seen, segmental resection is a sufficiently acceptable intervention in surgical procedure targeting the tumor, rather than peritumoral resection.

\section{Conclusion}

Intussusception is very rarely seen in adult patients. Compared to the idiopathic variant in observed in childhood, in almost all adult patients an underlying cause is found. GIST is a rare etiology in adult intussusception, and since the growth pattern does not usually suggest this possibility, this atypical presentation should be kept in mind in adult cases of intussusception.

\section{Conflict of Interest: None declared.}

Financial Disclosure: The authors declared that this study has received no financial support.

Authorship contributions: Concept-M.M.F.; Design - M.M.F.; Supervision - M.M.F.; Materials - M.M.F., N.E.B; Data collection \&/or processing - M.M.F., A.T.F., N.E.B, B.S.A.N.; Literature search - M.M.F.; Writing - M.M.F., S.T.; Critical review - A.T.F.

\section{REFERENCES}

1. Azar T, Berger DL. Adult intussusception. Ann Surg 1997;226:134-8.

2. Begos DG, Sandor A, Modlin IM. The diagnosis and management of adult intussusception. Am J Surg 1997;173:88-94.

3. Sornmayura P. Gastrointestinal stromal tumors (GISTs): a pathology view point. J Med Assoc Thai 2009;92:124-35.

4. Wang LT, Wu CC, Yu JC, Hsiao CW, Hsu CC, Jao SW. Clinical entity and treatment strategies for adult intussusceptions: 20 years' experience. Dis Colon Rectum 2007;50:1941-9.

5. Ghaderi H, Jafarian A, Aminian A, Mirjafari Daryasari SA. Clinical presentations, diagnosis and treatment of adult intussusception, a 20 years survey. Int J Surg 2010;8:318-20.

6. Jai SR, Bensardi F, Chehab F, Khaiz D, Bouzidi A. Jejunal lipoma with intermittent intussusception revealed by partial obstructive syndrome. Saudi J Gastroenterol 2008;14:206-7.

7. Vasiliadis K, Kogopoulos E, Katsamakas M, Karamitsos E, Tsalikidis C, Pringos B, et al. Ileoileal intussusception induced by a gastrointestinal stromal tumor. World J Surg Oncol 2008;6:133.

9. Gupta A, Gupta S, Tandon A, Kotru M, Kumar S. Gastrointestinal stromal tumor causing ileo-ileal intussusception in an adult patient a rare presentation with review of literature. Pan Afr Med J 2011;8:29.

9. Hoshino N, Murata T, Oka K, Kawakami K, Hoshino K, Sekoguchi $\mathrm{S}$, et al. Gastrointestinal stromal tumors of the small intestine that expressed c-kit protein. Intern Med 2000;39:914-9.

10. Nilsson B, Sjölund K, Kindblom LG, Meis-Kindblom JM, Bümming P, Nilsson $\mathrm{O}$, et al. Adjuvant imatinib treatment improves recurrence-free survival in patients with high-risk gastrointestinal stromal tumours (GIST). Br J Cancer 2007;96:1656-8.

11. Dematteo RP, Ballman KV, Antonescu CR, Maki RG, Pisters PW, Demetri GD, et al; American College of Surgeons Oncology Group (ACOSOG) Intergroup Adjuvant GIST Study Team. Adjuvant imatinib mesylate after resection of localised, primary gastrointestinal stromal tumour: a randomised, double-blind, placebo-controlled trial. Lancet 2009;373:1097-104.

12. Joensuu H, Eriksson M, Sundby Hall K, Hartmann JT, Pink D, Schütte J, et al. One vs three years of adjuvant imatinib for operable gastrointestinal stromal tumor: a randomized trial. JAMA 2012;307:1265-72. 